\title{
Modulation of Muscle Responses Evoked by Transcranial Magnetic Stimulation During the Acquisition of New Fine Motor Skills
}

\author{
ALVARO PASCUAL-LEONE, NGUYET DANG, LEONARDO G. COHEN, JOAQUIM P. BRASIL-NETO, \\ ANGEL CAMMAROTA, AND MARK HALLETT \\ Human Cortical Physiology Unit, Human Motor Control Section, Medical Neurology Branch, National Institute of \\ Neurological Disorders and Stroke, National Institutes of Health, Bethesda, Maryland 20892
}

\section{SUMMARY AND CONCLUSIONS}

1. We used transcranial magnetic stimulation (TMS) to study the role of plastic changes of the human motor system in the acquisition of new fine motor skills. We mapped the cortical motor areas targeting the contralateral long finger flexor and extensor muscles in subjects learning a one-handed, five-finger exercise on the piano. In a second experiment, we studied the different effects of mental and physical practice of the same five-finger exercise on the modulation of the cortical motor areas targeting muscles involved in the task.

2. Over the course of 5 days, as subjects learned the one-handed, five-finger exercise through daily 2 -h manual practice sessions, the cortical motor areas targeting the long finger flexor and extensor muscles enlarged, and their activation threshold decreased. Such changes were limited to the cortical representation of the hand used in the exercise. No changes of cortical motor outputs occurred in control subjects who underwent daily TMS mapping but did not practice on the piano at all (control group 1).

3. We studied the effect of increased hand use without specific skill learning in subjects who played the piano at will for $2 \mathrm{~h}$ each day using only the right hand but who were not taught the fivefinger exercise (control group 2) and who did not practice any specific task. In these control subjects, the changes in cortical motor outputs were similar but significantly less prominent than in those occurring in the test subjects, who learned the new skill.

4. In the second experiment, subjects were randomly assigned to a physical practice group, a mental practice group, or a control group. Subjects in each practice group physically or mentally practiced the five-finger piano exercise independently for $2 \mathrm{~h}$ daily for 5 days. The control group did not practice the exercise. All subjects had daily TMS mapping of the cortical motor areas targeting the long finger flexor and extensor muscles.

5 . Over the course of 5 days, mental practice alone led to significant improvement in the performance of the five-finger exercise, but the improvement was significantly less than that produced by physical practice alone. However, mental practice alone led to the same plastic changes in the motor system as those occurring with the acquisition of the skill by repeated physical practice.

6. We conclude that acquisition of the motor skills needed for the correct performance of a five-finger piano exercise is associated with modulation of the cortical motor outputs to the muscles involved in the task. This rapid modulation may occur through an increase of synaptic efficacy in existing neural circuits (long-term potentiation) or unmasking of existing connections due to disinhibition.

7. Mental practice alone seems to be sufficient to promote the modulation of neural circuits involved in the early stages of motor skill learning. This modulation not only results in marked performance improvement but also seems to place the subjects at an advantage for further skill learning with minimal physical practice.

\section{INTRODUCTION}

Playing the piano demands orderly, sequential control of individual finger movements and a high degree of bimanual coordination. Even when given information about the hand position, the finger motions, the sequence of keys to press, and the duration and velocity of each key press, a novice would still be unable to play even the simplest piano sonata. The piano student must understand the demands of the task, develop a cognitive representation of it, and initiate a first, centrally guided response. At first, his or her limbs will move slowly, with fluctuating accuracy and speed, and success will require visual, proprioceptive, and auditory feedback. With practice, the pianist can refine each single movement, link the different movements with the desired timing, and attain stability and fluency in the ordered sequence. Only then can the pianist shift his or her attention from the mechanical details of the performance to its artistic interpretation.

Acquisition of new fine motor skills may demand modification of the nervous system to accommodate the new procedures (Jenkins et al. 1990; Kaas 1991; Merzenich et al. 1990). The primary motor cortex (M1) may have a central role in skill learning considering the flexibility in the associations of sensory inputs to M1 neurons and in the associations between M1, spinal neurons, and somatic musculature (Aou et al. 1992; Iriki et al. 1989; Kaas 1991). Studies using noninvasive imaging and neurophysiological techniques have demonstrated skill-associated nervous system plasticity in humans. In blind subjects, for example, proficient Braille reading is associated with enlargement of the cortical sensorimotor representation of the reading finger (Pascual-Leone et al. 1993; Pascual-Leone and Torres 1993), and in normal subjects, learning of a complicated sequence of voluntary finger movements is associated with changes in the slow cortical DC potentials (Niemann et al. 1991) and transient increases in regional cercbral blood flow in the cerebellum and striatum (Seitz et al. 1990).

In the present study we used transcranial magnetic stimulation (TMS) to study the cortical motor areas targeting the contralateral long finger flexor and extensor muscles in subjects learning a one-handed, five-finger exercise on the piano. In a second experiment we studied the different effects of mental and physical practice on the acquisition of the 
same five-finger exercise and on the modulation of the cortical motor areas targeting muscles involved in the task.

\section{METHODS}

\section{Subjects}

We used different subjects for each experiment. In experiment 1 , we studied 18 subjects who were randomly assigned to a test group or one of two control groups. Each group consisted of three men and three women who were matched for age (mean age, 44 yr; range, 38-51 yr). In experiment 2, we studied 15 subjects who were randomly assigned to a physical practice group, a mental practice group, or a control group. Each group consisted of three men and two women who were matched for age (mean age, $32 \mathrm{yr}$; range, 19-42 yr). All subjects were right-handed, as determined by the Oldfield questionnaire (Oldfield 1971). None of the subjects had experience playing the piano or any other musical instrument. None of them knew how to typewrite using all fingers. None of them had jobs or were involved in daily activities that demanded skilled, fine-finger movements. All subjects had normal findings on neurological and general physical examinations. The protocolwas approved by the Institutional Review Board, and all subjects gave their written informed consent for the study. The transcranial magnetic stimulator was used under an Investigational Device Exemption from the Food and Drug Administration.

\section{Experiment 1}

In experiment 1, subjects performed the one-handed, five-finger exercise on a Yamaha electronic piano keyboard interfaced with a Macintosh IIci computer. The exercise consisted of the following sequence of finger movements (and notes): thumb (C), index finger (D), middle finger (E), ring finger $(F)$, little finger $(G)$, ring finger $(F)$, middle finger $(E)$, index finger $(D)$. A metronome marked a rhythm of 60 beats per minute, and the subjects were asked to match the thumb and little finger movements to the beat, intercalating the movements of the other fingers between the beats. The subjects were asked to try to perform the sequence of finger movements fluently, without pauses, and without skipping any key, and to pay particular attention to keeping the interval between the individual key presses constant and the duration and velocity of each key press the same.

On day 1, baseline TMS mapping of the cortical motor areas targeting the long finger flexor and extensor muscles bilaterally was done according to the technique described below. Subjects were then randomly assigned to a test group or to one of two control groups. Only subjects in the test group were taught the five-finger exercise. Thereafter, they practiced the exercise for $2 \mathrm{~h}$ daily on days $1-5$, had their performance tested, rested for 20-30 min, and had TMS mapping of the motor cortex.

The performance was tested by recording 20 sequential repetitions of the exercise on the computer with the use of a sequencing software package (Vision, Opcode, Menlo Park, CA) that allowed the analysis of the exact sequence of key presses, the interval between key presses, and the duration and velocity of each key press. Any key pressed out of order was considered an error, so more than one error was possible during each sequence. The anticipated interval between key presses was $0.25 \mathrm{~s}$ given that a rhythm of 60 beats per minute was marked by a metronome for each four intervals. After each daily test, the subjects were given feedback about their performance, as well as tips on how to improve.

Control group 1 had daily TMS mapping only. Control group 2 played the piano at will for $2 \mathrm{~h}$ each day with the use of only the right hand, rested for 20-30 min, and had daily TMS mapping. Subjects in control group 2 could play anything they wanted on the piano but were asked to press only one key at a time, therefore executing individual finger movements in self-generated sequences. They were asked not to repeat a given sequence. On day 5 , control groups 1 and 2 were also taught the exercise, and their performance on the test was recorded as for the test subjects.

All practice sessions were performed in the laboratory and were supervised by one of the investigators. Subjects were asked not to rehearse the task at home.

\section{Experiment 2}

In experiment 2, all subjects were taught the same five-finger exercise as in experiment 1. Baseline TMS mapping of their cortical motor areas targeting the long finger flexor and extensor muscles was done according to the technique described below. Thereafter, the subjects were randomly assigned to a physical practice group, a mental practice group, or a control group.

Subjects in each practice group physically or mentally practiced the exercise independently for $2 \mathrm{~h}$ daily for 5 days. The control group did not practice the exercise but had daily TMS mapping. Subjects were asked not to rehearse the task at home. During the practice session, subjects in the physical practice group were encouraged to repeatedly perform the exercise on the keyboard and were free to select their own strategy. Subjects in the mental practice group were asked to sit in front of the piano and try to visualize their fingers performing the exercise and to imagine the sound. They were not allowed to touch the piano keys or to rehearse the exercise by moving the fingers in the air. To assure that they were indeed not moving their fingers, electromyographic (EMG) activity from the long finger flexor and extensor muscles was monitored continually with the use of pairs of surface EMG electrodes taped to the skin over the belly of the muscle and displaying the activity on an oscilloscope at a sensitivity of $20 \mu \mathrm{V} /$ div. After the 1 st $10-15 \mathrm{~min}$, all subjects were able to follow the instructions without difficulty. The mental practice group had a single 2-h physical practice session at the end of day 5 . The results of task performance and TMS mapping following this physical practice session are reported as day $5^{\prime}$.

The performance of all subjects was tested daily by 20 sequential repetitions of the exercise in the same fashion as described for experiment 1. TMS mapping was performed 20-30 min after the test. Therefore performance assessment and TMS mapping were

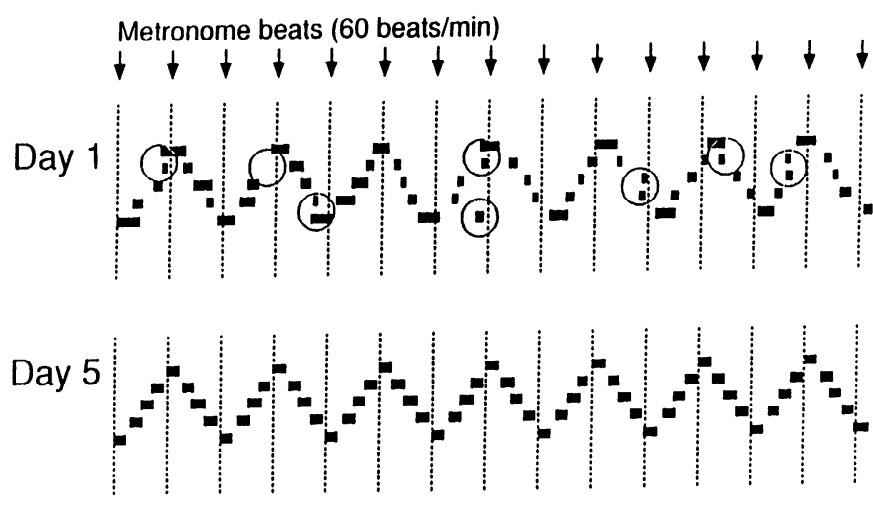

FIG. 1. Experiment 1: example of the performance in the 5-finger exercise on days 1 and 5 in a representative test subject. Arrows and dashed lines mark the metronome beats. Bars illustrate the different notes (finger movements) with the thumb being the lowest row of bars and the little finger the highest. The length of the bar represents the duration of each key press. Circles highlight the errors in the sequence performance. Comparison between days 1 and 5 illustrate the large number of errors, the highly variable key press durations, and the frequent deviations from the metronome on day 1 . 

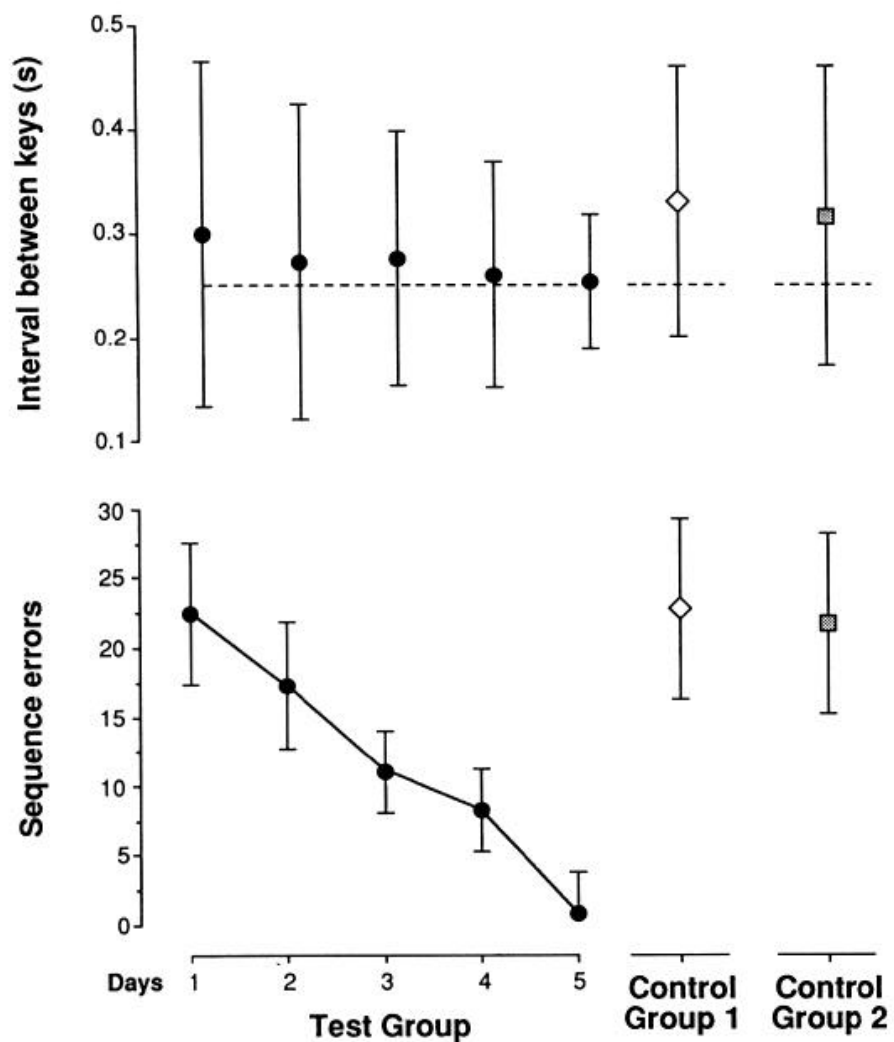

FIG. 2. Experiment 1: interval between key presses and number of sequence errors for all subjects (mean $\pm \mathrm{SD}$ ) in the 20-sequence test (5finger piano exercise) over the course of 5 days' learning. Dashed line indicates the anticipated interval of $0.25 \mathrm{~s}$ between key presses (see text for details).

the same in all three groups, which differed only in the learning conditions.

\section{Transcranial magnetic stimulation and mapping technique}

We used a Cadwell MES-10 magnetic stimulator equipped with an 8-shaped coil, which was held flat on the scalp with the intersection of its two "wings" centered over a defined scalp position; the handle of the coil was held horizontally, tangentially to the subject's head, pointing occipitally. This technique allows relatively focal stimulation (Cohen et al. 1990; Maccabee et al. 1990). The brain structures stimulated might be inferred from models of the induced electric fields (Roth et al. 1991a,b; Tofts 1990). However, this approximation might be affected by the existence of bends in the course of fibers that lower their threshold (Maccabee et al. 1993 ). In any case, overlay of TMS maps onto the magnetic resonance image of the subject's brain suggests that the motor responses are evoked from activation of the primary motor cortex (Levy et al. 1991; Wassermann et al. 1992).

For cortical mapping, scalp positions $1 \mathrm{~cm}$ apart were stimulated successively, following the technique described by Wassermann et al. (1991), and we calculated contour maps of the probability of evoking a motor potential with a peak-to-peak amplitude of at least $50 \mu \mathrm{V}$ in the contralateral muscles according to the stimulated scalp position. Eight single stimuli were given at each scalp position. On average, a stimulus was delivered every $3-5 \mathrm{~s}$. Each contour map represents 25 scalp positions ( $1 \mathrm{~cm}$ apart) arranged in a $5 \times 5$ grid around the "optimal" scalp position, which was marked on the subject's scalp with indelible ink. The mark, which remained throughout the 5-day experiment, was used as a reference point for the daily mapping. The optimal scalp position was the one from which TMS elicited motor evoked potentials (MEPs) of maximal amplitude in the contralateral finger flexor or extensor muscles. We have previously shown that optimal scalp positions determined in this manner project onto the posterior bank of the precentral sulcus and seem to represent activation of the primary motor cortex (Wassermann et al. 1992). The stimulus intensity was $110 \%$ of the subject's threshold on each particular day. Motor threshold was defined by the method of limits. In each subject we determined threshold as the average result from six series of stimuli, three with ascending stimulation intensities and three with descending stimulation intensities. The order of these six series of stimuli was randomly varied across subjects. For the ascending series we began stimulation at an intensity at which TMS did not evoke any identifiable MEPs in 10 trials. Thereafter, we increased the stimulation intensity in steps of $1 \%$ of the stimulator output. At each new intensity we applied 10 stimuli. Threshold intensity was defined as the lowest stimulation intensity at which TMS evoked $\geq 5$ MEPs of $\geq 50-\mu \mathrm{V}$ peak-to-peak amplitude. For the descending series we first stimulated at an intensity at which TMS evoked MEPs of $\geq 50 \mu \mathrm{V}$ peak-to-peak amplitude in 10 of 10 trials. Thereafter, we decreased the stimulation intensity in steps of $1 \%$ of the stimulator output. At each new intensity we applied 10 stimuli. Threshold intensity was defined as the lowest stimulation intensity at which
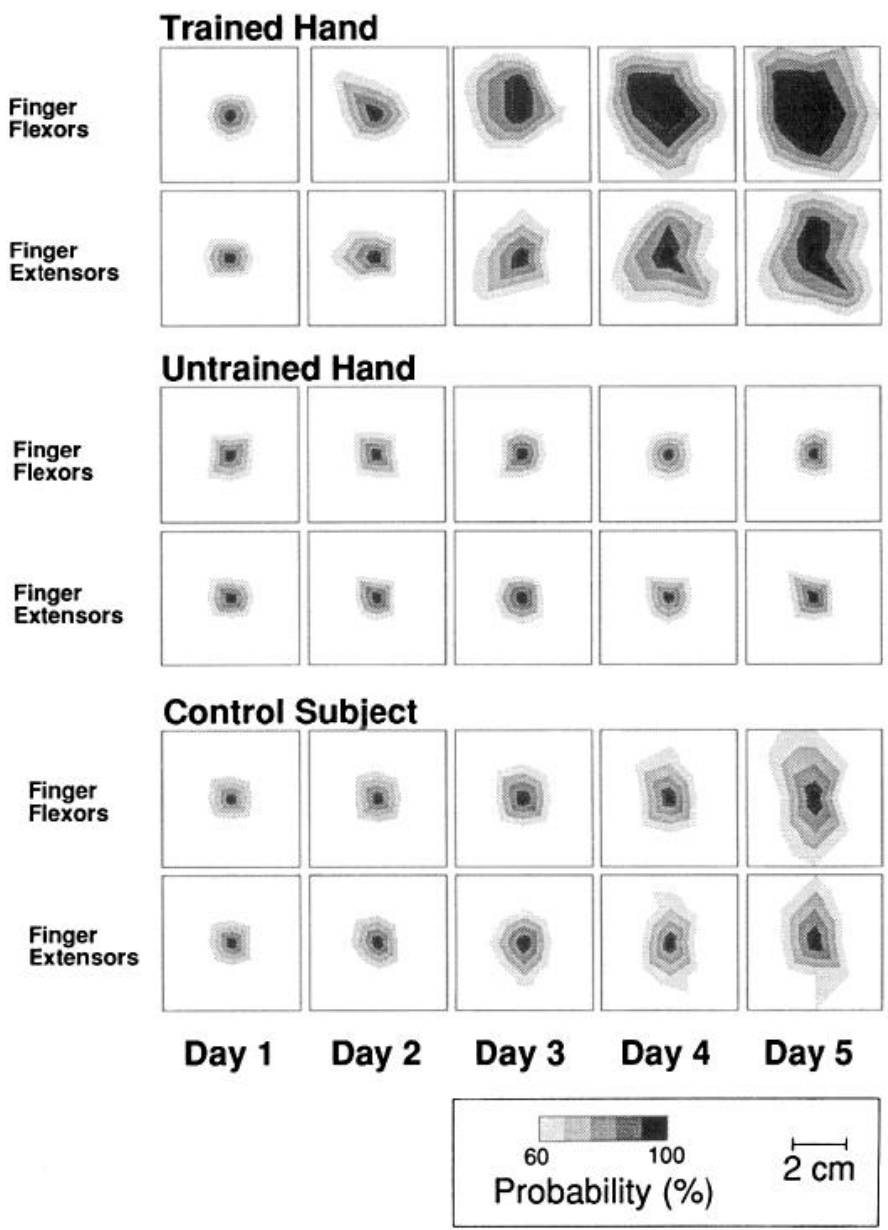

FIG. 3. Experiment 1: representative examples of the cortical motor output maps for the long finger flexor and extensor musles on days $1-5$ in a test subject (trained and untrained hand) and a subject from control group 2. Each map is based on 25 measured points. 


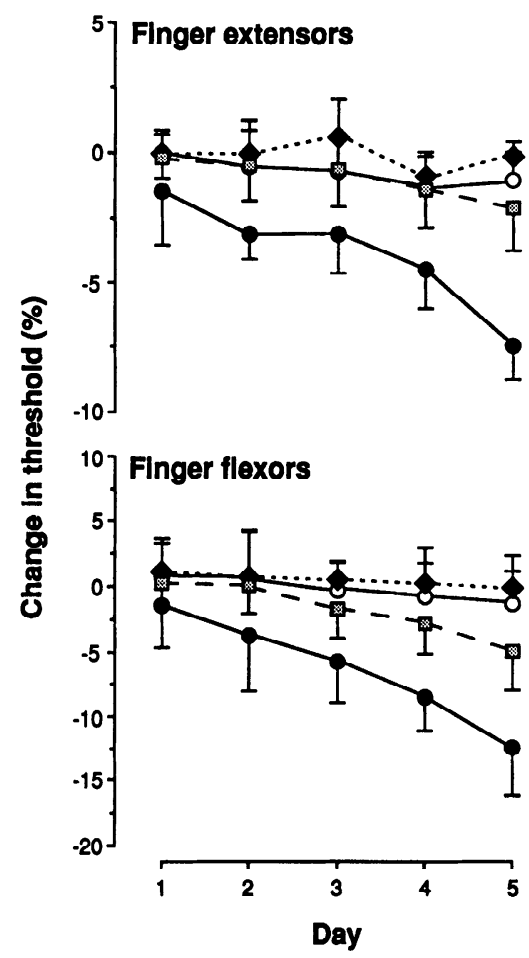

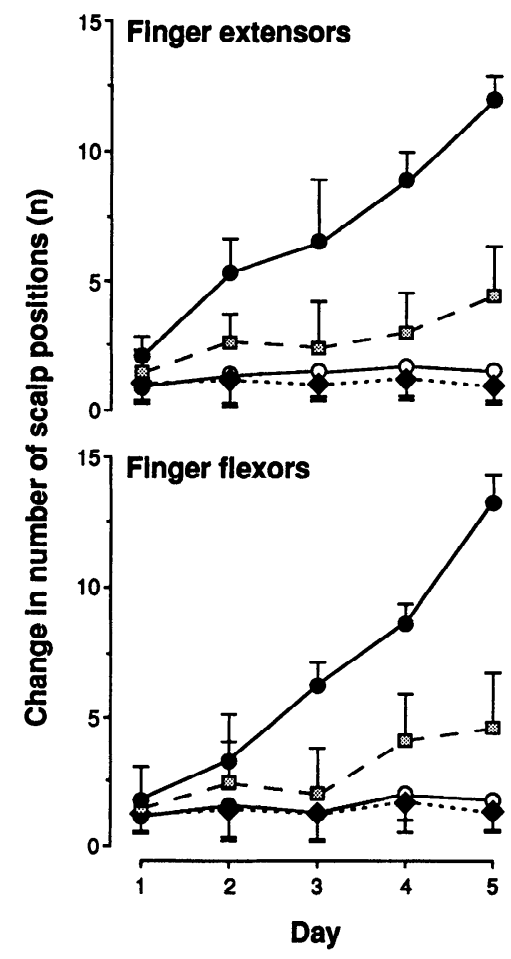

FIG. 4. Experiment 1: change in motor threshold, expressed as a percentage of change from baseline threshold, and in number of scalp positions on the stimulation grid from which motor evoked potentials (MEPs) could be produced in the finger flexor and extensor muscles with a probability of $\geq 60 \%$ in all subjects over the course of 5 days. Values are given as means \pm SD. Filled circles, trained hand of test subjects; open circles, untrained hand of test subjects; filled diamonds, control group 1; stippled squares, control group 2.
TMS evoked $\geq 5 \mathrm{MEPs}$ of $\geq 50-\mu \mathrm{V}$ peak-to-peak amplitude. Therefore the threshold in any given session represents the average value from six separate determinations and expresses the lowest stimulus intensity that evoked from the optimal scalp position motor potentials with a peak-to-peak amplitude of at least $50 \mu \mathrm{V}$ in $\geq 50 \%$ of the trials.

Mapping was performed with the subjects at rest. The absence of spontaneous, background EMG activity was documented by continuous EMG monitoring that was presented to the subjects by loudspeaker and on an oscilloscope at $20 \mu \mathrm{V}$ per division. Subjects were instructed to relax the target muscles completely achieving auditory silence and a flat line on the oscilloscope. EMG activity was recorded with a Dantec Counterpoint electromyograph with the use of pairs of surface EMG electrodes taped to the skin over the belly of the superficial finger flexor and extensor muscles in the forearm. This recording setup was not selective for movements of any particular digit but rather allowed monitoring of flexionextension activity of all fingers.

In both experiments the experimenter performing the TMS mapping was unaware of the group to which a given subject belonged in order to avoid biasing of the results.

\section{RESULTS}

\section{Experiment 1}

Over the course of the 5 days, the test group's playing skill improved markedly (Figs. 1 and 2). By day 5, the number of errors in the sequence of key presses clearly decreased, and the subjects correctly completed at least 18 of the 20 sequential repetitions of the exercise. The variability of the interval between key presses decreased, as illustrated by the narrowing of the standard deviation. The improvement in accuracy is illustrated by the decrease in the mean interval between key presses to $0.25 \mathrm{~s}$, which corre- sponds with the interval specified by the rhythm of 60 beats per minute marked by the metronome.

Concurrently with this improvement in performance, the threshold for activation of the finger flexor and extensor muscles contralateral to the side of TMS decreased steadily over the course of the 5 days, but only for the hand being trained. In addition, the size of the cortical output map for both muscle groups increased (Figs. 3 and 4 ). The increase in the size of the maps was independent of the threshold changes, because daily mapping of each muscle was performed at $110 \%$ of the subject's threshold on that day, thus controlling the effects of threshold changes across days.

As predicted, skill learning did not take place in the control subjects. Control groups 1 and 2 performed the exercise on day 5 with the same accuracy as the test subjects on day 1 (Fig. 2). In control group 1 , daily motor mapping with TMS did not affect the threshold for activation of the finger flexor and extensor muscles or the size of their cortical representation (Fig. 4). In control group 2, increased daily use of the hand by random piano playing resulted in a slight decrease in the threshold for TMS activation of the finger flexor and extensor muscles and in some increase in the size of the cortical representation for both muscle groups (Figs. 3 and 4). However, these changes were significantly smaller $[P<0.001$, analysis of variance (ANOVA)] than in the test subjects.

\section{Experiment 2}

Over the course of 5 days, both practice groups showed progressive improvement in their playing skills, as illustrated by a decrease in the number of sequence errors and a reduction in the variability (standard deviation) of the interval 
between key presses (Fig. 5). Accuracy increased in all practice subjects, as illustrated by a decrease in the mean interval between key presses to $0.25 \mathrm{~s}$. However, the physical practice group showed a significantly greater reduction $(P<$ 0.001 , ANOVA) in the number of sequence errors and a trend toward greater accuracy than did the mental practice group. The control group's performance did not improve.

Concurrently with the improvement in performance, the threshold for activation of the finger flexor and extensor muscles by TMS to the contralateral scalp decreased steadily over the course of the 5 days in the physical and mental practice groups. In addition, even though the threshold decrease was taken into account, the size of the cortical representation for both muscle groups increased equally for both practice groups but did not increase for the control group (Figs. 6 and 7).

Therefore mental practice alone led to significant fine motor skill learning but did not result in as much performance improvement as physical practice alone. However, mental practice alone led to the same plastic changes in the motor system as those occurring with the acquisition of a skill by repeated physical practice. By the end of day 5, the changes in the cortical motor outputs to the muscles involved in the
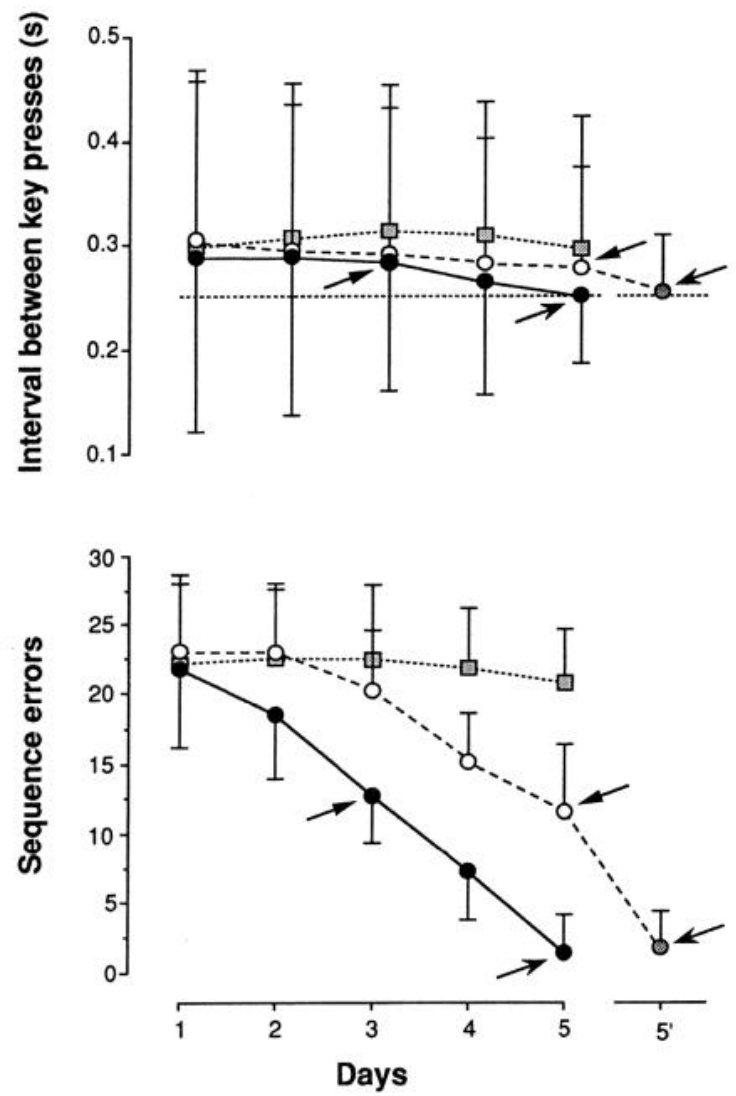

FIG. 5. Experiment 2: interval between key presses and number of sequence errors for all subjects (mean $\pm \mathrm{SD}$ ) in the 20-sequence test (5finger piano exercise) over the course of 5 days' learning. Dashed line indicates the anticipated interval between key presses $(0.25 \mathrm{~s})$. Arrows indicate the level of performance between the physical and mental practice groups at the end of day 5 or day $5^{\prime}$ ( see text for details). Filled circles, physical practice group; open circles, mental practice group; stippled squares, control group; stippled circle, mental practice group on day $5^{\prime}$.
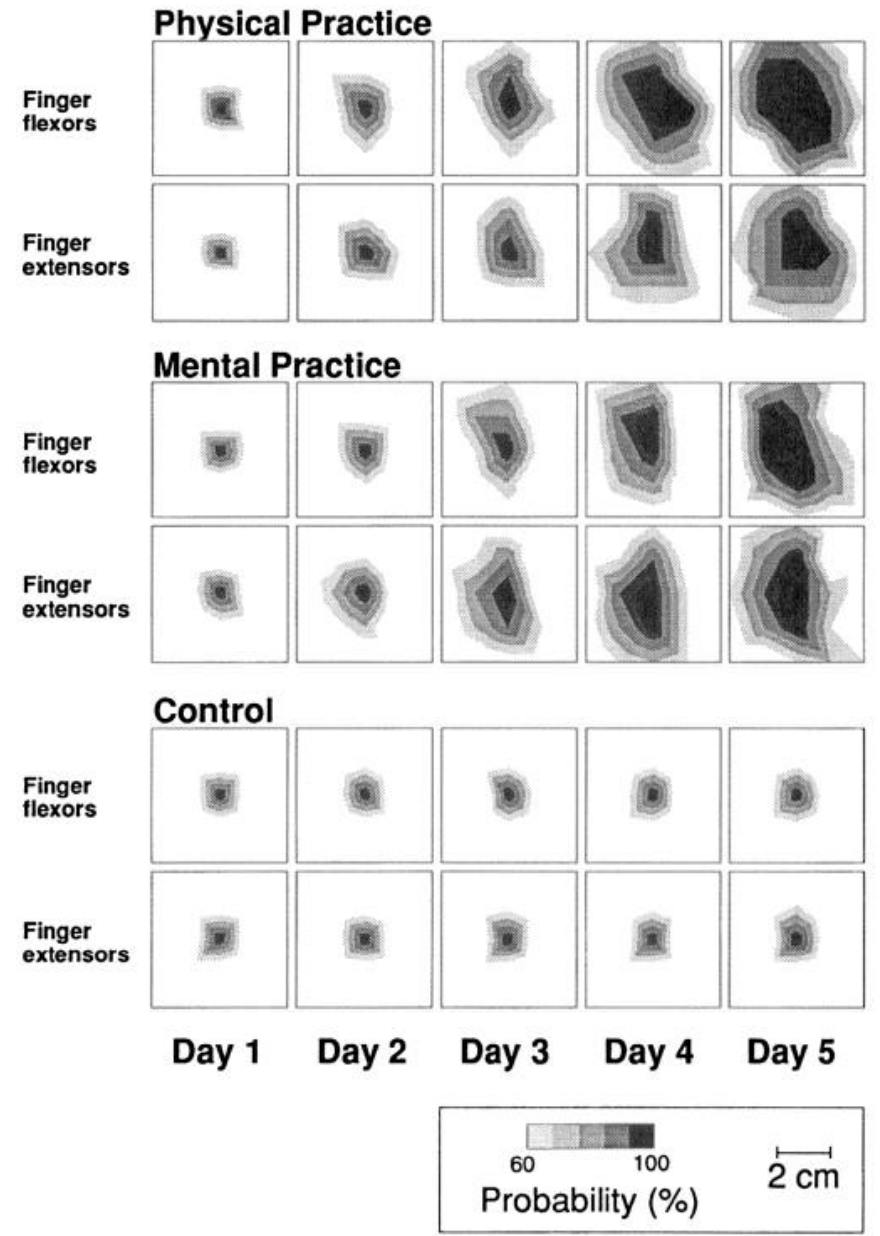

FIG. 6. Experiment 2: representative examples of the cortical motor output maps for the long finger flexor and extensor muscles on days 1-5 in a subject from each group. Each map is based on 25 measured points.

task did not differ between the physical and the mental practice groups (Fig. 5). However, the mental practice group's performance was at the level of that occurring with only 3 days' physical practice. After a single 2 -h physical practice session, the mental practice group's performance improved to the level of 5 days' physical practice (Fig. 5).

\section{DISCUSSION}

\section{Neurophysiological correlates of motor skill acquisition}

Recently, studies using noninvasive neurophysiological and imaging techniques have suggested that even the adult human nervous system is capable of reorganizing after injury, with the probable purpose of minimizing deficits and producing recovery of function (Cohen et al. 1991b, 1993; Pascual-Leone et al. 1992). These findings confirm in humans the growing body of evidence suggesting the flexible nature of connections in the nervous system of adult animals (Kaas 1991).

The nervous system of animals may also undergo changes according to the patterns of use, thereby providing a substrate for the acquisition of new skills (Jenkins et al. 1990; Merzenich et al. 1990). The sensorimotor representation of the pre- 


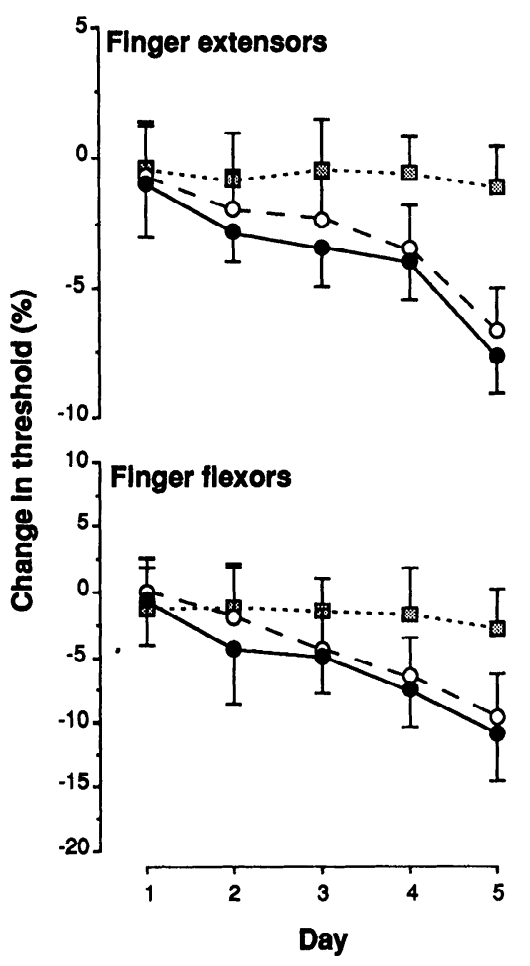

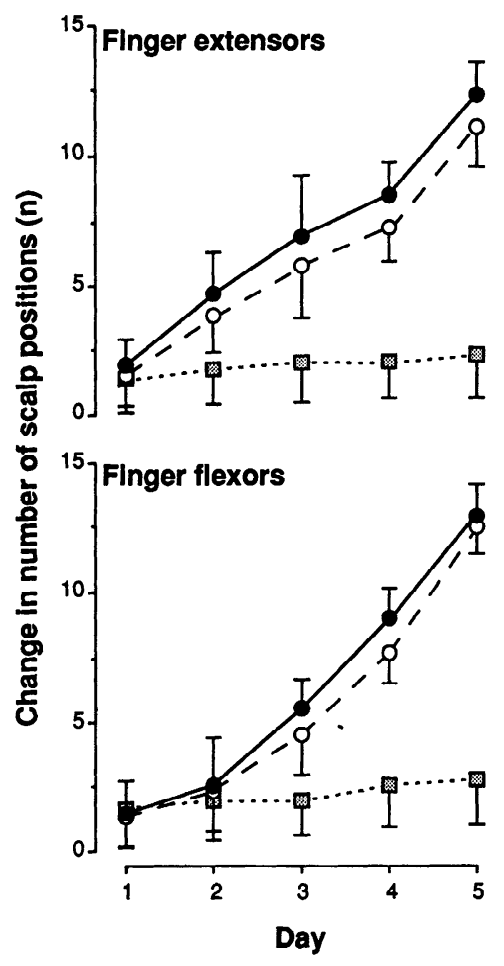

FIG. 7. Experiment 2: change in motor threshold, expressed as a percentage of the baseline threshold, and in number of scalp positions from which MEPs could be produced in the long finger flexor and extensor muscles with a probability of $\geq 60 \%$ in all subjects (mean $\pm \mathrm{SD}$ ) over the course of 5 days. Filled circles, physical practice group; open circles, mental practice group; stippled squares, control group. ferred hand in monkeys is more elaborate than that of the nonpreferred hand (Nudo et al. 1992), and training can result in distortions of body surface and movement representations that lead to behavioral gains (Jenkins et al. 1990). Motor cortical representation of a body part expands after selectively increased activity (Humphrey et al. 1990; Sanes et al. 1992), and differential stimulation of a restricted skin surface in a finger pad of adult monkeys leads to a reorganization of its somatosensory cortical representation, especially when stimulation has a functional significance (Recanzone et al. 1992a-d). In humans, a recent positron emission tomography study (Seitz et al. 1990) found that learning of a complicated sequence of voluntary finger movements is associated with increases in regional cerebral blood flow ( $\mathrm{rCBF}$ ) in the cerebellum and that acquisition of the motor skill results in an increase of $\mathrm{rCBF}$ in the striatum. Relative increases in rCBF have been shown to occur in the primary motor area, the supplementary motor area, and the thalamus as subjects learned a pursuit rotor task (Grafton et al. 1992). Finally, changes in slow cortical negative DC potentials during the acquisition of a complex finger motor task (Niemann et al. 1991) also suggest a dynamic modulation of the cortical representation of movement control during skill learning.

In the present study we found that acquisition of the motor skills needed for the correct performance of a five-finger piano exercise is associated with modulation of the cortical motor outputs to the muscles involved in the task. The cortical sensorimotor representation of a specific body part, as demonstrated, for example, by TMS mapping, may depend on the momentary level of excitability of the intracortical network that targets it. Extensive intracortical axonal collaterals provide inputs to many different movement representations of a given body part, and their pattern of recruitment may determine the execution of complex movements (Huntley and Jones 1991). In addition, neuronal networks targeting different body parts overlap widely and even share in part common neuronal elements (Schieber 1992). The neuronal elements in such networks may maintain a flexible balance based on demand and competition by their targets. Removal of a target, as in amputation or peripheral deafferentation, may result in the "takeover" of neuronal elements, formerly activated primarily as part of the network targeting the removed target, by neighboring networks targeting neighboring body parts. This would explain the reorganization of the motor outputs targeting muscles proximal to the stump of an amputated limb (Cohen et al. 1991a) or to an ischemic sensorimotor block (Brasil-Neto et al. 1992, 1993). Conversely, increased use and enhanced sensory feedback of a body part, especially if coupled with functional gain for the subject, may lead to a shift of the balance of intracortical networks toward that body part. Cortical networks underlying coordinated finger movements show a movement gradient across digits from the most prominently activated digit, which may depend on corticocortical or subcorticocortical projections (Amassian et al. 1989). Learning and practice may modulate the cortical outputs by strengthening such a gradient.

Modulation of motor cortical outputs may result from the establishment or unmasking of neuronal connections. The rapid time course of the motor output modulation, by which a certain region of motor cortex can increase its influence on a motoneuron pool, suggests the unmasking of existing neuronal connections, which may be due to decreased inhibition or increased synaptic efficacy (long-term potentiation) (Asanuma and Keller 1991; Iriki et al. 1989; Jacobs and Donoghue 1991). Such flexible modulation may represent a 
first stage in learning and could lead to structural changes in the intracortical and subcortical networks as the skill becomes overlearned and automatic. For example, Greenough et al. (Greenough 1984; Greenough et al. 1985) have shown that motor training is associated with changes in the dendritic branching patterns of motor and sensory cortical cells involved in the performance of the task. Sprouting may account for plastic changes in such situations, as likely occurred in monkeys deafferented for 10 years (Pons et al. 1991), and represents the correlate of long-standing "memories."

Learning-associated modulation of motor cortical outputs may result from changes in synaptic efficacy in the motor cortex itself (Jacobs and Donoghue 1991). However, our results do not allow ruling out that the observed plastic changes are driven by changes in the activity of other cortical areas or subcortical structures, for example, the premotor cortex or the cerebellum. Discharge patterns of Purkinje cells change during motor learning (Gilbert and Thach 1977; Ojakangas and Ebner 1992), which could lead to changes in cortical excitability via cerebellothalamocortical projections. A large proportion of cells in the premotor cortex in the monkey show a learning-dependent change in activity during the acquisition of visuomotor associations (Mitz et al. 1991), which could modulate primary motor cortex activity via corticocortical projections. Even a shift in the segmental excitability of appropriate spinal levels could account for the observed changes in the motor outputs. For example, an excitatory input to $\alpha$-motoneurons from suprasegmental levels or increased fusiform drive could bring the motoneurons close to firing level without overtly causing background EMG. Further studies are needed to define the level of the observed plasticity.

The changes in cortical motor outputs that we observed during TMS do not necessarily reflect the cortical activation pattern evoked during task performance, because TMS was applied while the subject was at rest, not performing the trained task. Therefore they must indicate a longer-lasting change possibly due to long-term synaptic potentiation (Asanuma and Keller 1991; Iriki et al. 1989; Jacobs and Donoghue 1991). We show that the "momentary level of excitability," a fixed time after performance of the task (20 min to $\sim 3 \mathrm{~h}$ ), rises progressively over the course of 5 days for the cortical outputs to muscles involved in the task as the subject's performance improves. Further practice may eventually lead to new changes in the cortical motor outputs as the task becomes overlearned and correct performance eventually may become "automatic" (Pascual-Leone et al. 1994). An important issue raised by our findings is the apparent lack of a plateau in the modulation of cortical outputs during learning. We believe that this apparent lack of plateau is likely an artifact of the duration of our study design. Our assumption would be that if we would continue with the training of the task used in the present study for longer than a week, there would be a plateau of the cortical output changes and even possibly a return of cortical thresholds to baseline as the skill becomes overlearned.

\section{Effect of manual and mental practice}

Mental practice is the imagined rehearsal of a motor act with the specific intent of learning or improving it, without overt movement output. Mental practice can be viewed as a virtual simulation of behavior by which the subject develops and "internally" rehearses a cognitive representation of the motor act. When confronted with a new motor task, the subject must develop a cognitive representation of it and initiate a centrally guided response, which secondarily can be improved by the use of sensorimotor feedback. Mental practice may accelerate the acquisition of a new motor skill by providing a well-suited cognitive model of the demanded motor act in advance of any physical practice (McBride and Rothstein 1979; Mendoza and Wichman 1978; White et al. 1979). Mental practice has found wide acceptance in the training of athletes (Denis 1985; Suinn 1984), and several famous instrumental musicians have used mental practice in the learning and rehearsal of new compositions (Schönberg 1987, 1988).

Studies of rCBF suggest that the prefrontal and supplementary motor areas, basal ganglia, and cerebellum are part of the network involved in the mental simulation of motor acts (Decety and Ingvar 1990; Ingvar and Philipson 1977; Roland et al. 1980, 1982, 1987; Roland and Friberg 1985). Therefore mental simulation of movements activates some of the same central neural structures required for the performance of the actual movements. In doing so, mental practice alone seems to be sufficient to promote the modulation of neural circuits involved in the early stages of motor skill learning. This modulation not only results in marked performance improvement but also seems to place the subjects at an advantage for further skill learning with minimal physical practice. The combination of mental and physical practice leads to greater performance improvement than physical practice alone (McBride and Rothstein 1979; White et al. 1979), a phenomenon for which our findings provide a physiological explanation. Mental imaging of movements recreates the effects of physical practice on the modulation of the central motor system and may, therefore, be an important adjunct not only for the learning of new motor skills but also for the maintenance of motor skills in temporarily immobilized patients and in the rehabilitation of patients with neurological disorders.

We thank B. J. Hessie for skillful editing.

Present addresses: A. Pascual-Leone, Unidad de Neurobiología, Departamento de Fisiología, Universidad de Valencia, Valencia 46010, Spain; J. P. Brasil-Neto, Laboratorio de Neurobiologia, Departamento de Ciencias Fisiologicas, Universidade de Brasilia, Brasilia 70910, Brazil.

Address for reprint requests: M. Hallett, Clinical Director, NINDS, Bldg. 10, Rm. 5N226, National Institutes of Health, Bethesda, MD 20892.

Received 21 April 1993; accepted in final form 28 April 1995.

\section{REFERENCES}

Amassian, V. E., Cracco, R. Q., and Maccabee, P. J. Focal stimulation of the human cerebral cortex with magnetic coil: a comparison with electrical stimulation. Electroencephalogr. Clin. Neurophysiol. 74: 401 416, 1989.

AOU, S. A., Woody, C. D., AND BIRT, D. Increases in excitability of neurons of the motor cortex of cats after rapid acquisition of eye blink conditioning. J. Neurophysiol. 12: 560-569, 1992.

AsANuma, H. AND Keller, A. Neuronal mechanisms of motor learning in mammals. Neuroreport 2: 217-24, 1991.

Brasil-Neto, J. P., Cohen, L. G., Pascual-Leone, A., Jabir, F. K., Wall, R. T., AND HALLETT, M. Rapid reversible reorganization in human motor 
system following transient deafferentation. Neurology 42: 1302-1306, 1992.

Brasil-Neto, J. P., Valls-Solé, J., Pascual-Leone, A., Cammarota, A., Amassian, V. E., Cracco, R., Maccabee, P., Cracco, J., Hallett, M., AND CoHEN, L. G. Rapid modulation of human cortical motor outputs following ischaemic nerve block. Brain 116: 511-525, 1993.

Cohen, L. G., Bandinelli, S., Findlay, T. W., and Hallett, M. Motor reorganization after upper limb amputation in man. Brain 114: 615-627, 1991 a.

Cohen, L. G., Brasil-Neto, J. P., Pascual-Leone, A., and Hallett, M. Plasticity of cortical motor output organization following deafferentation, cerebral lesions, and skill acquisition. Adv. Neurol. 63: 187-200, 1993.

Cohen, L. G., Roth, B. J., Nilsson, J., Dang, N., Bandinelli, S., Panizza, M., Friauf, W., AND Hallett, M. Effects of coil design on delivery of focal magnetic stimulation. Technical considerations. Electroencephalogr. Clin. Neurophysiol. 75: 350-357, 1990.

Cohen, L. G., Roth, B. J., Wassermann, E. M., Topka, H., Fuhr, P., SCHULTZ, J., AND HALLETT, M. Magnetic stimulation of the human cerebral cortex, an indicator of reorganization in motor pathways in certain pathological conditions. J. Clin. Neurophysiol. 8: 56-65, $1991 \mathrm{~b}$.

DECETY, J. AND INGVAR, D. H. Brain structures participating in mental simulation of motor behavior: a neuropsychological interpretation. Acta Psychol. 73: 13-34, 1990.

DENIS, M. Visual imagery and the use of mental practice in the development of motor skills. Can. J. Appl. Sport Sci. 10: 4S-16S, 1985.

GILBERT, P. F. C. AND THACH, W. T. Purkinje cell activity during motor learning. Brain Res. 128: 309-328, 1977.

Grafton, S. T., Mazziotta, J. C., Presty, S., Friston, K. J., Frackowiak, R. S. J., AND PHLEPS, M. E. Functional anatomy of human procedural learning determined with regional cerebral blood flow and PET. J. Neurosci. 12: 2542-2548, 1992.

GREENOUGH, W. T. Structural correlates of information storage in the mammalian brain: a review and hypothesis. Trends Neurosci. 7: 229-233, 1984.

Greenough, W. T., LARSON, J. R., AND Withers, G. S. Effects of unilateral and bilateral training in a reaching task on dendritic branching of neurons in the rat motor-sensory forelimb cortex. Behav. Neural Biol. 44: 301$314,1985$.

Humphrey, D. R., Oiu, X. O., Clavel, P., And O'Donoghue, D. L. Changes in forelimb motor representation in rodent cortex induced by passive movements Soc. Neurosci. Abstr. 16: 422, 1990.

HunTLEY, G. W. AND JoNES, E. G. Relationship of intrinsic connections to forelimb movement representations in monkey cortex: a correlative anatomic and physiological study. J. Neurophysiol. 66: 390-413, 1991

Ingvar, D. H. AND Philipson, L. Distribution of the cerebral blood flow in the dominant hemisphere during motor ideation and motor performance. Ann. Neurol. 2: 230-237, 1977.

IrIKı, A., Pavlides, C., Keller, A., AND AsANUma, H. Long-term potentiation of motor cortex. Science Wash. DC 245: 1385-1387, 1989.

JACOBS, K. M. AND DONOGHUE, J. P. Reshaping the cortical motor map by unmasking latent intracortical connections. Science Wash. DC 251: 944$947,1991$.

Jenkins, W. M., Merzenich, M. M., and Recanzone, G. Neocortical representational dynamics in adult primates: implications for neuropsychology. Neuropsychologia 28: 573-584, 1990.

KAAS, J. H. Plasticity of sensory and motor maps in adult mammals. Annu. Rev. Neurosci. 14: 137-167, 1991.

Levy, W. J., Amassian, V. E., Schimd, U. D., AND Jungreis, C. Mapping of motor cortex gyral sites non-invasively by transcranial magnetic stimulation in normal subjects and patients. In: Magnetic Motor Stimulation: Basic Principles and Clinical Experience, edited by W. J. Levy, R. Q. Cracco, A. T. Barker, and J. Rothwell. Amsterdam: Elsevier, 1991, p. $51-75$.

Maccabee, P. J., Amassian, V. E., Eberle, L., AND Cracco, R. Q. Magnetic coil stimulation of straight and bent amphibian and mammalian peripheral nerve in vitro: locus of excitation. J. Physiol. Lond. 460: 201219, 1993.

Maccabee, P. J., Eberle, L., Amassian, V. E., Cracco, R. Q., Rudell, A. P., AND JAYACHANDRA, M. Spatial distribution of the electric field induced in volume by a round and figure ' 8 ' magnetic coils: relevance to activation of sensory nerve fibers. Electroencephalogr. Clin. Neurophysiol. 76: 131-141, 1990.
McBride, E. R. AND Rothstein, A. L. Mental and physical practice and the learning and retention of open and closed skills. Percept. Mot. Skills 49: 359-365, 1979.

Mendoza, D. W. AND Wichman, H. 'Inner' darts: effects of mental practice on performance of dart throwing. Percept. Mot. Skills 47: 1195-1199, 1978.

Merzenich, M. M., Recanzone, G. H., Jenkins, W. M., and Grajski, K. A. Adaptive mechanisms in cortical networks underlying cortical contributions to learning and nondeclarative memory. Cold Spring Harbor Symp. Quant. Biol. 55: 873-887, 1990.

Mitz, A. R., GodSCHALK, M., AND WISE, S. P. Learning-dependent neuronal activity in the premotor cortex: activity during the acquisition of conditional motor associations. J. Neurosci. 11: 1855-1872, 1991.

Niemann, J., Winker, T., Gerling, J., LANDwehrmeyer, B., AND Jung, $R$. Changes in slow cortical negative DC-potentials during the acquisition of a complex finger motor task. Exp. Brain Res. 85: 417-422, 1991.

Nudo, R. J., Jenkins, W. M., Merzenich, M. M., Prejean, T., And GRENDA, R. Neurophysiological correlates of hand preference in primary motor cortex of adult squirrel monkeys. J. Neurosci. 12: 2918-2947, 1992.

OJakangas, C. L. and Ebner, T. J. Purkinje cell complex and simple spike changes during a voluntary arm movement learning task in the monkey. J. Neurophysiol. 68: 2222-2236, 1992.

OldFiELD, R. C. The assessment and analysis of handedness: the Edinburgh inventory. Neuropsychologia 9: 97-113, 1971.

Pascual-Leone, A., Cammarota, A., Wassermann, E. M., Cohen, L. G., AND HALLETT, M. Modulation of motor cortical outputs to the reading hand of Braille readers. Ann. Neurol. 34: 33-37, 1993.

Pascual-Leone, A., Cohen, L. G., AND Hallett, M. Noninvasive evaluation of plastic changes in human motor system. Trends Neurosci. 15: 13-15, 1992.

Pascual-Leone, A., Grafman, J., AND Hallett, M. Modulation of cortical motor output maps during the development of implicit and explicit knowledge. Science Wash. DC 263: 1287-1289, 1994.

PASCUAL-LeONE, A. AND TORRES, F. Plasticity of the sensorimotor cortex representation of the reading finger in Braille readers. Brain 116: 39$52,1993$.

Pons, T. P., Garraghty, P. E., Ommaya, A. K., Kass, J. H., Taub, E., AND MishKIN, M. Massive cortical reorganization after sensory deafferentation in adult macaques. Science Wash. DC 252: 1857-1860, 1991.

Recanzone, G. H., Jenkins, W. M., HradeK, G. T., and Merzenich, M. M. Progressive improvement in discriminative abilities in adult owl monkeys performing a tactile frequency discrimination task. J. Neurophysiol. 67: 1015-1030, 1992a.

Recanzone, G. H., Merzenich, M. M., and Jenkins, W. M. Frequency discrimination training engaging a restricted skin surface results in an emergence of a cutaneous response zone in cortical area 3a. J. Neurophysiol. 67: 1057-1070, 1992b.

Recanzone, G. H., Merzenich, M. M., Jenkins, W. M., Grajski, K. A., AND DINSE, H. R. Topographic reorganization of the hand representation in cortical area $3 \mathrm{~b}$ owl monkeys trained in a frequency-discrimination task. J. Neurophysiol. 67: 1031-1056, 1992c.

Recanzone, G. H., Merzenich, M. M., ANd Schreiner, C. E. Changes in the distributed temporal response properties of SI cortical neurons reflect improvements in performance on a temporally based tactile discrimination task. J. Neurophysiol. 67: 1071-1091, 1992d.

Roland, P. E., Ericksson, L., Stone-Elander, S., and Widen, L. Does mental activity change the oxidative metabolism of the brain? J. Neurosci. 7: 2373-2389, 1987.

ROLAND, P. E. AND FRIBERG, L. Localization of cortical areas activated by thinking. J. Neurophysiol. 53: 1219-1243, 1985.

Roland, P. E., LaRSEN, B., Lassen, N. A., AND Skinhoj, E. Supplementary motor area and other cortical areas in organization of voluntary movements in man. J. Neurophysiol. 43: 118-136, 1980.

Roland, P. E., Meyer, E., Shibasaki, T., Yamamoto, Y. L., And Thompson, C. J. Regional cerebral blood flow changes in cortex and ganglia during voluntary movements in normal human volunteers. J. Neurophysiol. 48: 467-478, 1982.

Roth, B. J., Cohen, L. G., AND Hallett, M. The electric field induced during transcranial magnetic stimulation. Electroencephalogr. Clin. Neurophysiol. Suppl. 43: 268-278, 1991a.

Roth, B. J., Saypol, J. M., Hallett, M., AND Cohen, L. G. A theoretical 
calculation of the electric field induced in the cortex during magnetic stimulation. Electroencephalogr. Clin. Neurophysiol. 81: 47-56, 1991b.

SAnes, J. N., WANG, J., AND Donoghue, J. P. Immediate and delayed changes of rat motor cortical output representation with new forelimb configurations. Cereb. Cortex 2: 141-152, 1992.

SCHIEBER, M. H. Widely distributed neuron activity in primary motor cortex area during individuated finger movements. Soc. Neurosci. Abstr. 18: $504,1992$.

SChOnberg, H. Great Pianists. St. Louis, MO: Fireside Books, 1987. SCHONBERG, H. The Virtuosi. New York: Vintage-Random House, 1988.

Seitz, R. J., Roland, E., Bohm, C., Greitz, T., and Stone-Elander, S. Motor learning in man: a positron emission tomographic study. Neuroreport 1: 57-60, 1990.
SuINN, R. M. Imagery and sport. In: Imagery and Sport, edited by W. F. Straub and J. M. Williams. Lansing, NY: Sport Science Associates, 1984.

ToFTS, P. S. The distribution of induced currents in magnetic stimulation of the nervous system. Phys. Med. Biol. 35: 1119-1128, 1990.

Wassermann, E. M., McShane, L. M., Hallett, M., and Cohen, L. G. Noninvasive mapping of muscle representations in human motor cortex. Electroencephalogr. Clin. Neurophysiol. 85: 1-12, 1991.

Wassermann, E. M., Wang, B., Toro, C., Zeffiro, T., Valls-Solé, J., Pascual-Leone, A., AND Hallett, M. Projecting transcranial magnetic stimulation (TMS) maps into brain MRI. Soc. Neurosci. Abstr. 18: 939, 1992.

White, C. A., Ashton, R., AND Lewis, S. Learning a complex skill: effect of mental practice, physical practice and imagery ability. Int. J. Sports Psychol. 10: 71-78, 1979. 